\title{
Plasma microRNA alterations between EGFR-activating mutational NSCLC patients with and without primary resistance to TKI
}

\author{
Yihan Ma ${ }^{1}$, Xiaoyan Pan ${ }^{1,2}$, Peiqi Xu ${ }^{3}$, Yanjun $\mathrm{Mi}^{1}$, Wenyi Wang ${ }^{1}$, Xiaoting Wu ${ }^{1}, Q^{i}$ \\ $\mathrm{He}^{1}$, Xinli Liu ${ }^{1}$, Weiwei Tang ${ }^{1}$ and Han-Xiang An ${ }^{1}$ \\ ${ }^{1}$ Xiamen Cancer Hospital the First Affiliated Hospital of Xiamen University, 361003 Fujian, China \\ ${ }^{2}$ Department of Medical Oncology, Linyi Cancer Hospital, 276000 Shandong, China \\ ${ }^{3}$ Reproduction Center, The Second Affiliated Hospital of Kunming Medical University, 650101 Yunnan, China \\ Correspondence to: Han-Xiang An, email: anhanxiang@xmu.edu.cn \\ Keywords: EGFR, EGFR-TKI, NSCLC, circulating miRNA, primary resistance \\ Received: November 02, $2016 \quad$ Accepted: July 18, $2017 \quad$ Published: August 03, 2017 \\ Copyright: Ma et al. This is an open-access article distributed under the terms of the Creative Commons Attribution License 3.0 \\ (CC BY 3.0), which permits unrestricted use, distribution, and reproduction in any medium, provided the original author and source \\ are credited.
}

\section{ABSTRACT}

Epidermal growth factor receptor (EGFR) tyrosine kinase inhibitors (TKIs) have obtained excellent therapeutic effects against non-small cell lung cancer (NSCLC) harboring activating EGFR mutations. However, some patients have exhibited primary resistance which becomes a major obstacle in effective treatment of NSCLC. The mechanisms of EGFR-TKIs resistance involved are still poorly understood. Many studies suggest that miRNAs play an important role in regulating drug sensitivity of EGFR-TKIs. The aim of the present study was to examine differentially expressed miRNAs in plasma between EGFR-TKIs sensitive and EGFR-TKIs primary resistance patients. MiRNA microarray of plasma from patients' blood identified 16 differentially expressed miRNAs of which 15 (hsv2-miR-H19, hsa-miR-744-5p, hsa-miR-3196, hsa-miR-3153, hsa-miR-4791, hsa-miR-4803, hsa-miR-4796-3p, hsa-miR-372-5p, hsa-miR-138-2-3p, hsa-miR-16-1-3p, hsa-miR-1469, hsa-miR-585-3p, ebv-miRBART14-5p, hsa-miR-769-3p, hsa-miR-548aq-5p) were down regulated while only hsa-miR-503-3p was up regulated in primary resistant patients' plasma. Volcano plot and hierarchical clustering were performed to examine the accuracy of the miRNAs. Then validation with quantitative real-time PCR was performed and the result was in accordance with the array data. Functional analysis of these differentially expressed miRNAs with Ingenuity Pathway Analysis (IPA) revealed a common signaling network including MYC, CCND1, IGF1 and RELA. In conclusion, our finding may play important role in understanding the mechanisms underlying the problem and should be further evaluated as potential biomarkers in primary resistance of NSCLC.

\section{INTRODUCTION}

Lung cancer is the worldwide leading cause of cancer-related death [1]. It is classified into NSCLC and small cell lung cancer (SCLC) by histological category. More than $80 \%$ of lung cancers are NSCLC, which includes adenocarcinoma, squamous cell carcinoma, large cell carcinoma, and bronchioalveolar carcinoma. Chemotherapy treatment and other treatment regime didn't bring ideal effect and were far from satisfaction [2]. With recent developments of targeted therapy, EGFR -TKIs had achieved huge success and became one of the standard treatment-regimes of NSCLC [3]. It had been proved that the effectiveness of EGFR-TKIs was superior to chemotherapy in advanced NSCLC with activating EGFR mutations [4]. However, the efficiency of EGFR-TKIs is increasingly limited by drug resistance, approximately $10 \%$ of patients with EGFR-activating mutations do 
not exhibit objective responses to EGFR TKIs from the beginning (primary resistance). Although possible mechanisms like PTEN/PI3K/AKT pathway and NF-кB have been investigated in some studies, vital explanations are still missing [5].

MiRNAs are small ( 22-nucleotides long), non-coding, single-stranded RNAs that regulate gene expression post-transcriptionally. They are responsible for various biological and pathological processes including cancer development and progression [6]. In recent years, the dysregulation of miRNAs has been highlighted as one of the mechanisms underlying primary resistance of TKIs. It has been also proved that several miRNAs enhance therapeutic efficacy to EGFR-TKIs by modulating the sensitivity of cancer cells [7].

In our study, we compared plasma miRNA expressions between EGFR-TKI primary resistance patients and EGFR-TKIs sensitive patients. We hope that the profiles can provide a new perspective into the problem and further serve as a potential therapeutic approach to overcoming EGFR-TKIs primary resistance in NSCLC.

\section{RESULTS}

\section{Differential miRNA expression profiles}

According to data processing and analysis, specific miRNAs were identified to be differentially expressed between EGFR-TKI primary resistance patients and EGFR-TKI sensitive patients. The analysis revealed 16 miRNAs among which 15 miRNAs were down-regulated (hsv2-miR-H19, hsa-miR-744-5p, hsa-miR-3196, hsamiR-3153, hsa-miR-4791, hsa-miR-4803, hsa-miR-47963p, hsa-miR-372-5p, hsa-miR-138-2-3p, hsa-miR-16-1-3p, hsa-miR-1469, hsa-miR-585-3p, ebv-miR-BART14-5p, hsa-miR-769-3p, hsa-miR-548aq-5p) while only hsamiR-503-3p was up-regulated in EGFR-TKI primary resistance patients. Differentially expressed miRNAs were selected by volcano plot filtering (fold change $\geq 1.5$ and $\mathrm{P}$-value $\leq 0.05$ ) as shown in Figure 1 . There were 1 upregulated miRNA and 15 down-regulated miRNAs as the fold numbers presented in Table 1 . Then we performed hierarchical clustering of these miRNAs. We found these miRNAs clearly discriminated the EGFR-TKI primary resistance patients from EGFR-TKI sensitive patients, as shown in Figure 2.

\section{Validation with quantitative real-time PCR}

The expression levels of miRNAs were identified in 8 Plasma samples by RT-qPCR. As shown in Figure 3, Hsv2-miR-H19, hsa-miR-744-5p, hsa-miR-3196, hsamiR-3153, hsa-miR-4791, hsa-miR-4803, hsa-miR-47963p, hsa-miR-372-5p, hsa-miR-138-2-3p, hsa-miR-16-1-3p, hsa-miR-1469, hsa-miR-585-3p, ebv-miR-BART14-5p, hsa-miR-769-3p, hsa-miR-548aq-5p were down regulated while only hsa-miR-503-3p was up regulated in primary resistant patients' plasma.

\section{Network and molecular function analyses}

16 miRNAs were mapped, network-eligible and classified into genetic network. 11 miRNAs at least share one overlapping gene in common as shown in Figure 4. IPA also depicted important biological pathway associated with this network. Meanwhile, different molecular events directly related to neoplasm were identified (i.e. cell death and survival, cellular development, cellular growth and proliferation). Furthermore, their relationships with diseases and disorders were also assessed.

The other 5 miRNAs were detected in separate networks without overlapping due to the lack of commonly-shared gene.

\section{DISCUSSION}

To investigate the possible mechanisms underlying primary resistance to EGFR-TKIs, we identified patients exhibiting primary resistance with NSCLC harboring EGFR activating mutations. We found several miRNA alterations in plasma related to primary resistance in a small subset of patients. The study well represented the demographic and clinicopathologic characteristics of patients with EGFR-mutant NSCLC, which could provide practical considerations for EGFR-TKIs treatment in the precision treatment era.

Our results show that a total of 16 plasma miRNAs were differentially expressed including miR-744-5p, miR138-2-5p, miR-3153, miR-4791, miR-4803, miR-4796-3p, miR-372-5p, miR-548aq-5p and hsv2-miR-H19. These miRNAs may play an important role in primary resistance to NSCLC with activating EGFR mutations. In addition, one network was detected and emerged via overlapping genes with the analysis of IPA concerning cell death and survival. Recent studies have reported on the role of miRNAs in EGFR-TKIs resistance. MiR-21 was verified to have significantly higher expression in resistant patients $[8,9]$. Our findings are in agreement with these papers that miRNAs play and important role in the mechanism underlying EGFR-TKIs resistance. Furthermore, we provided more new miRNAs which could possibly add new knowledge to the subject.

MYC exhibits extremely important functions in cell cycle progression, apoptosis and cellular transformation. Functional experiment showed that miR-744 suppresses tumor by targeting c-Myc which leads to the inhibition of HCC cell growth [10]. What's more, it has a more specific relationship with lung cancer and has been proved to take part in the enhanced glutamine metabolism which could possibly be a surrogate marker to predict the respond to EGFR-TKIs [11]. Its emergence here probably indicates the potential to be a biomarker or target. CCND1 is 
Table 1: Differentially expressed miRNAs

\begin{tabular}{lcc}
\hline Name & Fold change (resistance vs sensitivity) & P-value \\
\hline hsa-miR-503-3p & 3.399 & 0.041 \\
hsv2-miR-H19 & 0.374 & 0.004 \\
hsa-miR-744-5p & 0.410 & 0.011 \\
hsa-miR-3196 & 0.482 & 0.036 \\
hsa-miR-3153 & 0.389 & 0.016 \\
hsa-miR-4791 & 0.446 & 0.014 \\
hsa-miR-4803 & 0.351 & 0.048 \\
hsa-miR-4796-3p & 0.385 & 0.044 \\
hsa-miR-372-5p & 0.356 & 0.030 \\
hsa-miR-138-2-3p & 0.331 & 0.022 \\
hsa-miR-1469 & 0.492 & 0.018 \\
hsa-miR-585-3p & 0.476 & 0.003 \\
ebv-miR-BART14-5p & 0.280 & 0.003 \\
hsa-miR-769-3p & 0.465 & 0.013 \\
hsa-miR-548aq-5p & 0.452 & 0.010 \\
hsa-miR-16-1-3p & 0.477 & 0.010 \\
\hline
\end{tabular}

\section{Volcano Plot ( Treatment vs Control )}

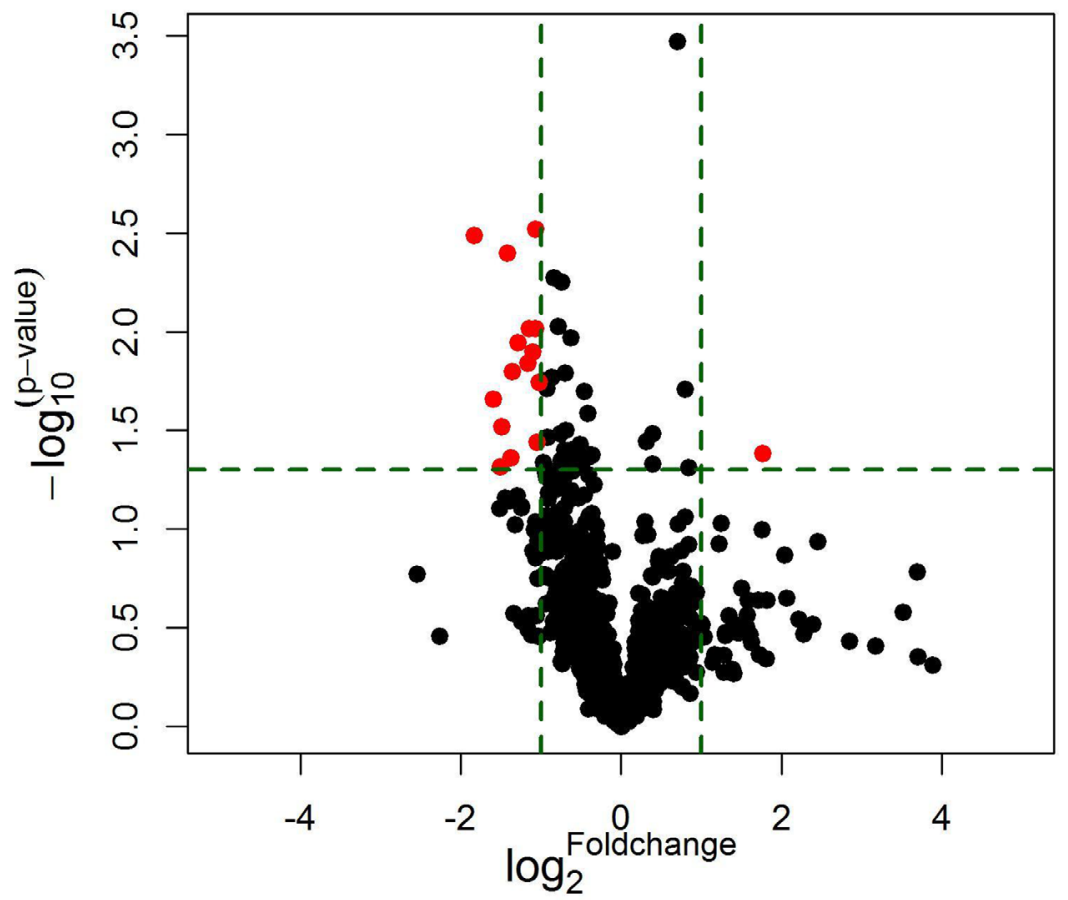

Figure 1: The vertical lines correspond to $\mathbf{2 . 0}$-fold up and down, respectively, and the horizontal line represents a p-value of $\mathbf{0 . 0 5}$. The red point in the plot represents the differentially expressed miRNAs with statistical significance. 16 miRNAs (red plots) passed the volcano plot filtering. 
reported to be a key target in NSCLC development and can be regulated by many different factors like mir-146a$5 \mathrm{p}$ and mir-134 PSAT1 [12-14]. Relevant mechanisms include causing cell cycle arrest at the G0/G1 phase and great difference is shown in inhibiting cell proliferation, migration, invasion, and promoting apoptosis by targeting CCND1. Besides, CCND1 has a close relationship with MYC so the function they perform together in this network may worth investigating. RELA stands for NF$\kappa \mathrm{B}$, a present in almost all cell types and is the endpoint of a series of signal transduction events such as cell growth, tumorigenesis and apoptosis. Recently a study showed that NF- $\kappa \mathrm{B}$ activation may replace the oncogenic EGFR signaling in NSCLC when EGFR is inhibited by TKI in the presence of the T790M [15]. Therefore, its inhibition may be a promising therapeutic option for those who progress after original EGFR-TKIs treatment. PI3K is originally involved in normal cellular functions which in turn involved in cancer. However, recently a growing body of evidence has shown that it could do more in NSCLC and TKI resistance especially take PTEN/PI3K/ AKT pathway into consideration [16]. Since clinical PI3K inhibition has been somewhat disappointing, people are now trying to co-inhibit PI3K with other factors at the same time to achieve a better outcome [17, 18]. MMP9 is closely associated with cancer, due to its role in extracellular matrix remodeling and angiogenesis. Although there are some paper mentioning its role in lung cancer, its place in TKI resistance remains largely unknown [19]. BCL2 and ERBB2 are mostly referred to in NSCLC but not TKI treatment. For example, BCL2 has been proved to be connected with resistance to platinumbased chemotherapy while ERBB2 is merely said to be involved in the tumorgenesis [20]. So, whether they could

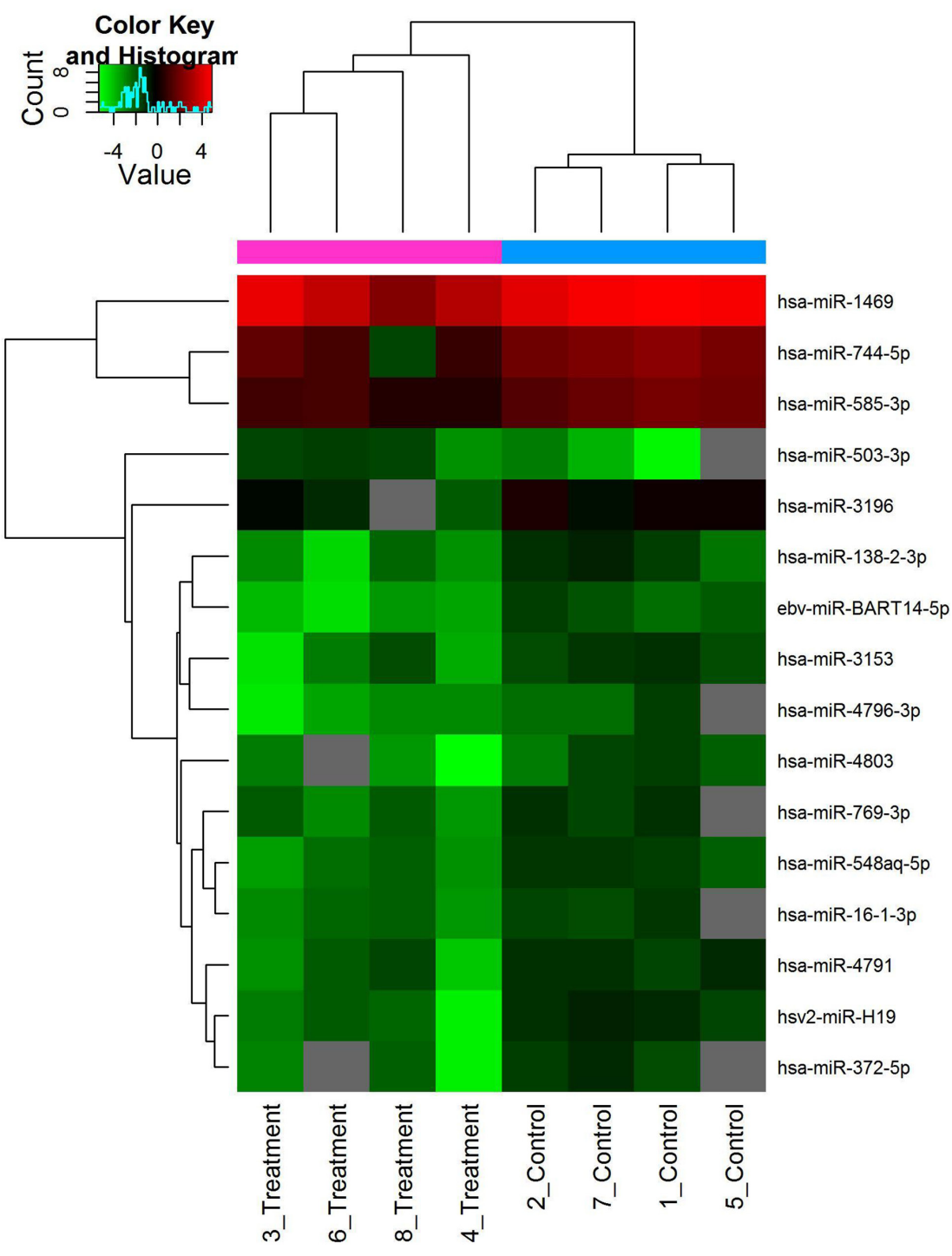

Figure 2: Hierarchical clustering of differentially expressed miRNAs. Scale bar: up-regulated (red), down-regulated (green). $\log 2$ transformed data were used. 


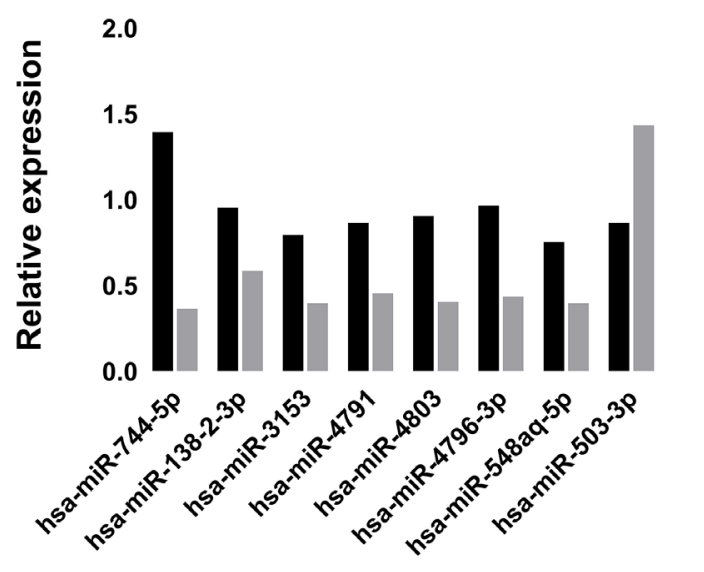

Sensitivity

Resistance

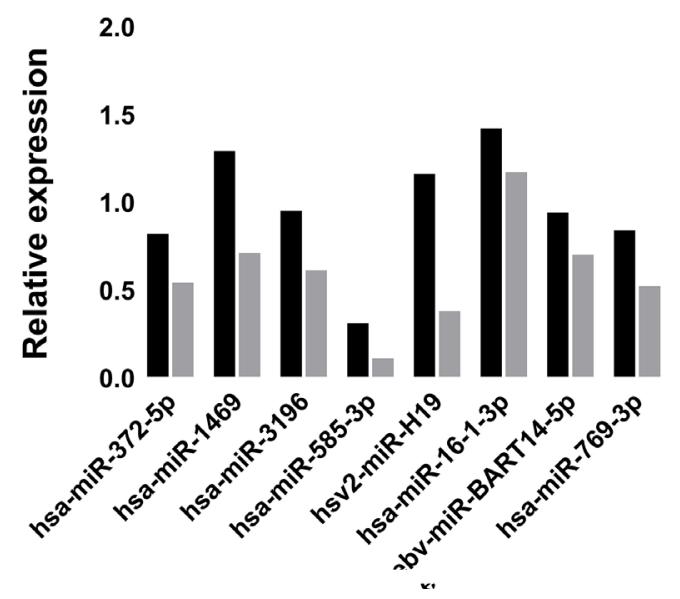

Figure 3: The expression of real-time qPCR was in accordance with microarray data.

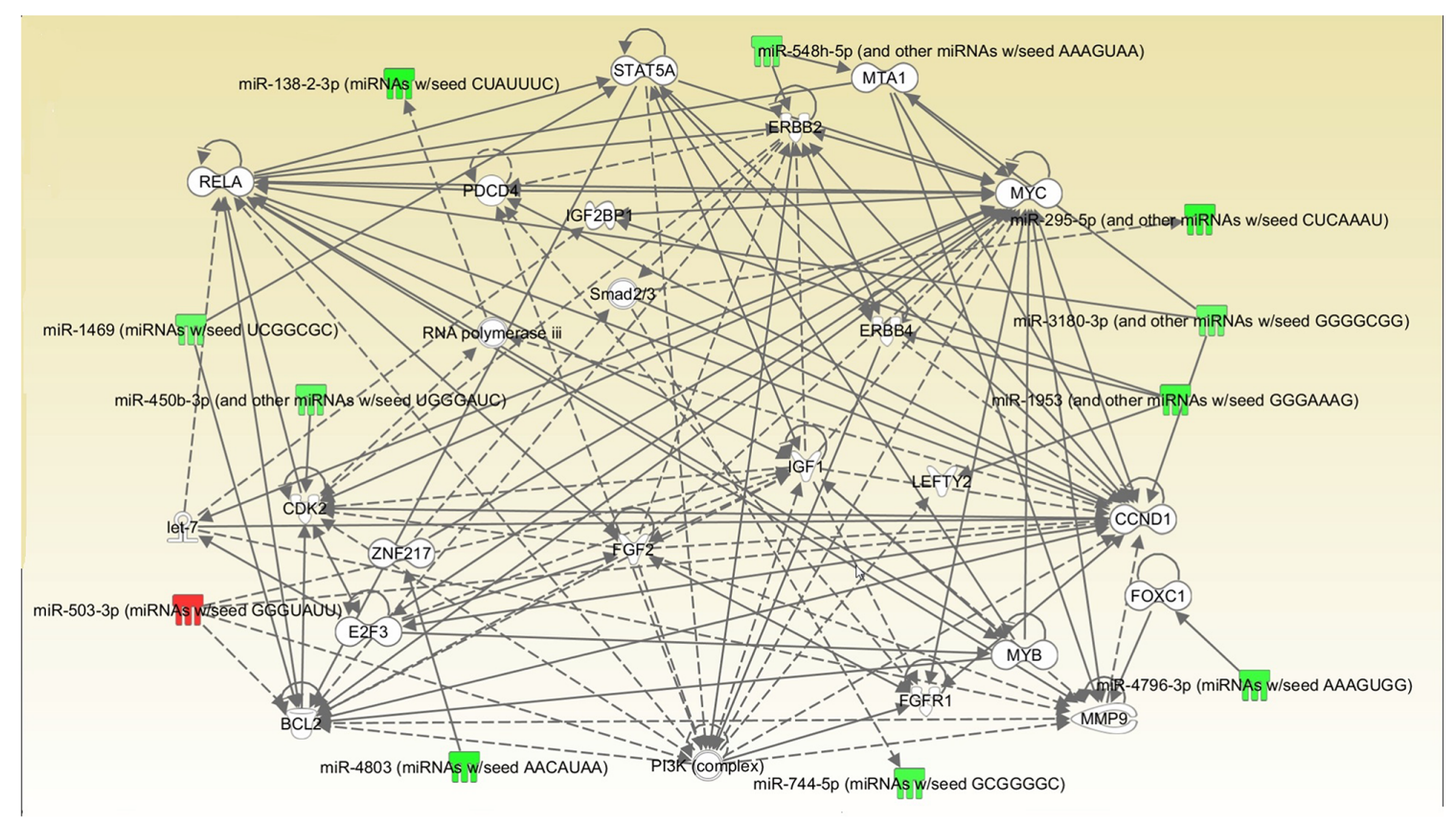

Figure 4: Interrelated networks of genes and miRNAs whose expression was different between TKI-sensitive patients and primary resistant ones. One important network of interrelated miRNAs and target genes were identified. $\mathrm{P}=0.036$. 
serve as potential target in overcoming primary resistance is still to be uncovered.

In conclusion, the emergence of key regulators such as MYC, CCND1 and RELA could be important in distinguishing the relevant mechanisms. Genetic mutation or deletion leads to the inactivation of tumor suppressor genes in the carcinogenesis. Furthermore, miRNAs modify tumor suppressor genes at transcriptional level and suppress their functions in response of TKI [21].

Surely the problem is still from fully understood. For example, ethnicity, T790M and other bypass mechanisms. Therefore, future studies are needed to examine these in detail and will provide insight into their roles in NSCLC TKI treatment.

\section{MATERIALS AND METHODS}

\section{Patients and samples collection}

Blood samples were collected from NSCLC patients with EGFR-activating mutations who received gefitinib or erlotinib between 2013 and 2015 treated in the Xiamen Cancer Hospital, the First Affiliated Hospital of Xiamen University. Blood samples were obtained with patients' informed consent. EGFR activating mutations were defined as mutations known to be associated with EGFR TKI sensitivity, including exon 19 deletion and L858R. Imaging data were reviewed to evaluate treatment responses according to the Response Evaluation Criteria in Solid Tumors recommended by the WHO, defined as complete remission (CR), partial remission (PR), stable disease (SD), and progressive disease (PD) on the basis of the published literature. We defined primary resistance as disease progression in $<3$ months (90 days) without any evidence of objective response while receiving EGFR TKI treatment. For each sample, $5-10 \mathrm{~mL}$ of blood was taken in EDTA-containing vacuum tubes. Plasma and blood cells were separated by centrifugation at $820 \times g$ for $10 \mathrm{~min}$ at $4^{\circ} \mathrm{C}$, Plasma was then centrifuged at $2500 \times \mathrm{g}$ for $10 \mathrm{~min}$ at $4^{\circ} \mathrm{C}$. Plasma was stored at $-80^{\circ} \mathrm{C}$.

\section{RNA extraction}

Plasma was separated within half an hour with two centrifuging steps $(850 \mathrm{~g} / 10 \mathrm{~min}$ and $2400 \mathrm{~g} / 10 \mathrm{~min})$ at $4^{\circ} \mathrm{C}$. Total RNA was isolated from $200 \mu \mathrm{l}$ plasma. Total RNA was isolated using TRIzol (Invitrogen) and purified with RNeasy mini kit (QIAGEN) according to manufacturer's instructions. RNA quality and quantity was measured by using nanodrop spectrophotometer (ND-1000, Nanodrop Technologies) and RNA Integrity was determined by gel electrophoresis.

\section{MiRNA labeling and array hybridization}

RNA labeling and array hybridization was according to Exiqon's manual. After quality control, the miRCURY'м Hy3 $^{\text {TM}} / \mathrm{Hy} 5^{\text {TM }}$ Power labeling kit (Exiqon, Vedbaek, Denmark) was used according to the manufacturer's guideline for miRNA labelling by following steps: $1 \mu \mathrm{LRNA}$ in $2.0 \mu \mathrm{L}$ of water was combined with $1.0 \mu \mathrm{L}$ of CIP buffer and CIP (Exiqon). The mixture was incubated for $30 \mathrm{~min}$ at $37^{\circ} \mathrm{C}$. The Reaction was terminated by incubation for $5 \mathrm{~min}$ at $95^{\circ} \mathrm{C}$. Then 3.0 $\mu \mathrm{L}$ of labeling buffer, $1.5 \mu \mathrm{L}$ of fluorescent label (Hy3TM), $2.0 \mu \mathrm{L}$ of DMSO, $2.0 \mu \mathrm{L}$ of labeling enzyme were added into the mixture. The labeling reaction was incubated for $1 \mathrm{~h}$ at $16^{\circ} \mathrm{C}$ Terminated by incubation for $15 \mathrm{~min}$ at $65^{\circ} \mathrm{C}$. After stopping the labeling procedure, the Hy3 $3^{\mathrm{TM}_{-}}$-labeled samples were hybridized on the miRCURYTM LNA Array (v.18.0) (Exiqon) according to array manual. The total $25 \mu \mathrm{L}$ mixture from Hy3 $3^{{ }^{\mathrm{M}}}$-labeled samples with 25 $\mu \mathrm{L}$ hybridization buffer were first denatured for $2 \mathrm{~min}$ at $95^{\circ} \mathrm{C}$, incubated on ice for $2 \mathrm{~min}$. Then hybridized to the microarray for $16-20 \mathrm{~h}$ at $56^{\circ} \mathrm{C}$ in a 12 -Bay Hybridization Systems (Hybridization System-Nimblegen Systems, Inc., Madison, WI, USA). Following hybridization, the slides were achieved, washed several times using Wash buffer kit (Exiqon). Then the slides were scanned using the Axon GenePix 4000B microarray scanner (Axon Instruments, Foster City, CA).

\section{Data processing and analysis}

Scanned images were then imported into GenePix Pro 6.0 software (Axon) for grid alignment and data extraction. Replicated miRNAs were averaged and miRNAs that intensities $>=30$ in all samples were chosen for calculating normalization factor. Expressed data were normalized using the Median normalization. After normalization, significant differentially expressed miRNAs between two groups were identified through Fold change and P-value. Differentially expressed miRNAs between two samples were filtered through Fold change. Finally, hierarchical clustering was performed to show distinguishable miRNA expression profiling among samples.

\section{Validation with quantitative real-time PCR (RT- qPCR)}

To validate microarray data, we analyzed the expression of miRNAs using quantitative Real TimePolymerase Chain Reaction (RT-qPCR). We used the miScript PCR System (Qiagen) for reverse transcription and RT-qPCR. RNA was converted into cDNA using the miScript II Reverse Transcription Kit and the HiSpec Buffer according to the manufacturers' protocol. The RTqPCR was performed with the miScript SYBR ${ }^{\circledR}$ Green PCR Kit in a total volume of $20 \mu \mathrm{L}$ per reaction containing $1 \mu \mathrm{L}$ diluted cDNA according to the manufacturers' protocol. MiR-191-5p served as endogenous control since this miRNA has been reported as a suitable normalizes of miRNA values in plasma. 


\section{Statistical analysis}

The comparison of different miRNA expression in plasma between primary resistant patients and sensitive patients was analyzed using the Students t-test. The Fisher's test was used to analyze the significance of the genetic networks identified by the IPA tool. A p value $<$ 0.05 was considered statistically significant.

\section{Network and gene ontology analyses}

Genetic networks and functional classification of differentially expressed miRNAs were investigated with IPA (Ingenuity Systems, Mountain View, CA), a web delivered tool that enables the discovery, visualization, and exploration of molecular interaction networks in gene expression data.

\section{ACKNOWLEDGMENTS}

None.

\section{CONFLICTS OF INTEREST}

The authors declare no conflicts of interests.

\section{GRANT SUPPORT}

This study was partially supported by a grant from the Fujian Provincial Health and Family Planning Commission Foundation of Youth Scientific Research Project (grant no. 2015-2-42) and Xiamen Science and Technology Bureau Foundation of Science and Technology Project for the benefit of the people (grant no.3502Z20164010).

\section{REFERENCES}

1. Siegel RL, Miller KD, Jemal A. Cancer statistics, 2015. CA Cancer J Clin. 2015; 65: 5-29. https://doi.org/10.3322/ caac. 21254.

2. Breathnach OS, Freidlin B, Conley B, Green MR, Johnson DH, Gandara DR, O'Connell M, Shepherd FA, Johnson BE. Twenty-two years of phase III trials for patients with advanced non-small-cell lung cancer: sobering results. J Clin Oncol. 2001; 19: 1734-42. https://doi.org/10.1200/ jco.2001.19.6.1734.

3. Ranson M. Epidermal growth factor receptor tyrosine kinase inhibitors. Br J Cancer. 2004; 90: 2250-5. https:// doi.org/10.1038/sj.bjc.6601873.

4. Sequist LV, Martins RG, Spigel D, Grunberg SM, Spira A, Janne PA, Joshi VA, McCollum D, Evans TL, Muzikansky A, Kuhlmann GL, Han M, Goldberg JS, et al. First-line gefitinib in patients with advanced non-smallcell lung cancer harboring somatic EGFR mutations. J
Clin Oncol. 2008; 26: 2442-9. https://doi.org/10.1200/ JCO.2007.14.8494.

5. Ohashi K, Maruvka YE, Michor F, Pao W. Epidermal growth factor receptor tyrosine kinase inhibitor-resistant disease. J Clin Oncol. 2013; 31: 1070-80. https://doi. org/10.1200/JCO.2012.43.3912.

6. Calin GA, Croce CM. MicroRNA signatures in human cancers. Nat Rev Cancer. 2006; 6: 857-66. https://doi. org/10.1038/nrc1997.

7. Sin TK, Wang F, Meng F, Wong SC, Cho WC, Siu PM, Chan LW, Yung BY. Implications of microRNAs in the treatment of gefitinib-resistant non-small cell lung cancer. Int J Mol Sci. 2016; 17: 237. https://doi.org/10.3390/ ijms 17020237.

8. Wang S, Su X, Bai H, Zhao J, Duan J, An T, Zhuo M, Wang Z, Wu M, Li Z, Zhu J, Wang J. Identification of plasma microRNA profiles for primary resistance to EGFR-TKIs in advanced non-small cell lung cancer (NSCLC) patients with EGFR activating mutation. J Hematol Oncol. 2015; 8: 127. https://doi.org/10.1186/s13045-015-0210-9.

9. Ricciuti B, Mecca C, Cenci M, Leonardi GC, Perrone L, Mencaroni C, Crino L, Grignani F, Baglivo S, Chiari R, Sidoni A, Paglialunga L, Curra MF, et al. miRNAs and resistance to EGFR-TKIs in EGFR-mutant non-small cell lung cancer: beyond 'traditional mechanisms' of resistance. Ecancermedicalscience. 2015; 9: 569. https:// doi.org/10.3332/ecancer.2015.569.

10. Lin F, Ding R, Zheng S, Xing D, Hong W, Zhou Z, Shen J. Decrease expression of microRNA-744 promotes cell proliferation by targeting c-Myc in human hepatocellular carcinoma. Cancer Cell Int. 2014; 14: 58. https://doi. org/10.1186/1475-2867-14-58.

11. Serizawa M, Kusuhara M, Zangiacomi V, Urakami K, Watanabe M, Takahashi T, Yamaguchi K, Yamamoto N, Koh Y. Identification of metabolic signatures associated with erlotinib resistance of non-small cell lung cancer cells. Anticancer Res. 2014; 34: 2779-87.

12. Li YL, Wang J, Zhang CY, Shen YQ, Wang HM, Ding L, Gu YC, Lou JT, Zhao XT, Ma ZL, Jin YX. MiR-146a-5p inhibits cell proliferation and cell cycle progression in NSCLC cell lines by targeting CCND1 and CCND2. Oncotarget. 2016; 7: 59287-98. https://doi.org/10.18632/ oncotarget.11040.

13. Yang Y, Wu J, Cai J, He Z, Yuan J, Zhu X, Li Y, Li M, Guan H. PSAT1 regulates cyclin D1 degradation and sustains proliferation of non-small cell lung cancer cells. Int J Cancer. 2015; 136: E39-50. https://doi.org/10.1002/ ijc. 29150 .

14. Sun CC, Li SJ, Li DJ. Hsa-miR-134 suppresses non-small cell lung cancer (NSCLC) development through downregulation of CCND1. Oncotarget. 2016; 7: 35960-78. https://doi.org/10.18632/oncotarget.8482.

15. Galvani E, Sun J, Leon LG, Sciarrillo R, Narayan RS, Sjin RT, Lee K, Ohashi K, Heideman DA, Alfieri RR, Heynen 
GJ, Bernards R, Smit EF, et al. NF- $\kappa$ B drives acquired resistance to a novel mutant-selective EGFR inhibitor. Oncotarget. 2015; 6: 42717-32. https://doi.org/10.18632/ oncotarget.3956.

16. Perez-Ramirez C, Canadas-Garre M, Molina MA, FausDader MJ, Calleja-Hernandez MA. PTEN and PI3K/AKT in non-small-cell lung cancer. Pharmacogenomics. 2015; 16: 1843-62. https://doi.org/10.2217/pgs.15.122.

17. Zhu X, Jiang H, Li J, Xu J, Fei Z. Anticancer effects of paris saponins by apoptosis and PI3K/AKT pathway in gefitinibresistant non-small cell lung cancer. Med Sci Monit. 2016; 22: $1435-41$.

18. Heavey S, Cuffe S, Finn S, Young V, Ryan R, Nicholson S, Leonard N, McVeigh N, Barr M, O’Byrne K, Gately $\mathrm{K}$. In pursuit of synergy: an investigation of the PI3K/ mTOR/MEK co-targeted inhibition strategy in NSCLC. Oncotarget. 2016; 7:79526-43. https://doi.org/10.18632/ oncotarget. 12755 .

19. Bai L, Lin G, Sun L, Liu Y, Huang X, Cao C, Guo Y, Xie C. Upregulation of SIRT6 predicts poor prognosis and promotes metastasis of non-small cell lung cancer via the ERK1/2/MMP9 pathway. Oncotarget. 2016; 7: 40377-86. https://doi.org/10.18632/oncotarget.9750.

20. Wang Y, Ha M, Liu J, Li P, Zhang W, Zhang X. Role of BCL2-associated athanogene in resistance to platinumbased chemotherapy in non-small-cell lung cancer. Oncol Lett. 2016; 11: 984-90. https://doi.org/10.3892/ ol.2015.4003.

21. Donzelli S, Cioce M, Muti P, Strano S, Yarden Y, Blandino G. MicroRNAs: non-coding fine tuners of receptor tyrosine kinase signalling in cancer. Semin Cell Dev Biol. 2016; 50: 133-42. https://doi.org/10.1016/j.semcdb.2015.12.020. 